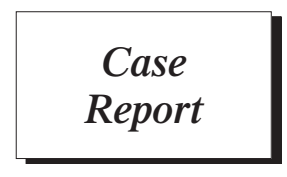

\title{
A Surgical Case Report of Off-pump Onlay Patch Grafting for Pseudoaneurysm with Diffusely Calcified Coronary Artery
}

\author{
Shiro Sasaguri, MD, Hideaki Nishimori, MD, Seiichiro Wariishi, MD, \\ Masaki Yamamoto, MD, Nobuo Kondo, MD, Kazuki Kihara, MD, and Takashi Fukutomi, MD
}

\begin{abstract}
Coronary pseudoaneurysm usually occurs after catheter-based intervention as a result of traumatic dissection or perforation of a coronary artery in $4 \%-5 \%$ of cases. Here, we report the successful case of pseudoaneurysm occurring after percutaneous coronary angioplasty (PTCA) against the severely calcified coronary artery treated with off-pump long onlay patch bypass technique using internal thoracic arteries. We report the case of a 59-year-old woman treated with off-pump onlay patch grafting for pseudoaneurysm after coronary angioplasty against the diffusely calcified coronary lesion. The portion of the endoarterectomized left anterior descending coronary artery proximal to the resected aneurysm extending $5 \mathrm{~cm}$ distally was successfully reconstructed with long onlay patch using the internal thoracic arterial graft.
\end{abstract}

Key words: off-pump, CABG, aneurysm, cardiac intervention, calcification

\section{Case Report}

Coronary pseudoaneurysm usually occurs after a catheter-based intervention as a result of traumatic dissection or perforation of a coronary artery, resulting in the disruption of the media without blood seepage through the adventitia occurring in $4 \%-5 \%$ of cases. ${ }^{1,2)}$ The patient is a 59-year-old female with poorly controlled diabetes mellitus and a history of peripheral arterial disease, who underwent post bilateral femoropopliteal bypass surgery. She presented with a recent history of unstable angina worsening over the last week. The emergent coronary

Department of Surgery II, Faculty of Medicine, Kochi University, Okoh-cho, Nankoku, Kochi, Japan

Received: July 27, 2009; Accepted: November 11, 2009

Corresponding author: Shiro Sasaguri, MD. Department of Surgery II, Faculty of Medicine, Kochi University, Okoh-cho, Nankoku, Kochi 783-8505, Japan

Email: sasaguri@kochi-u.ac.jp

(C)2011 The Editorial Committee of Annals of Thoracic and Cardiovascular Surgery. All rights reserved. angiography revealed diffusely diseased coronary vessels with $99 \%$ stenosis of calcified left anterior descending artery (LAD) and 90\% lesion of the circumflex artery (Fig. 1a). The target lesion of LAD was inflated with a $1.5-\mathrm{mm}$ balloon. The re-study performed at two weeks later revealed the aneurysm formation at the distal site of percutaneous coronary angioplasty (PTCA) (Fig. 1b), which was followed by three-dimensional coronary angiography with computed tomography (3D CT). The aneurysm showed a gradual increase in size up to $1 \mathrm{~cm}$ within 2 weeks, which indicated the necessity of surgical intervention (Fig. 2a).

The operation was performed using an off-pump long onlay bypass technique that is almost similar to the method described by Takanashi et al. ${ }^{2)}$ The surgery was performed according to the institutional review board guidelines, and informed consent was obtained from the patient. In brief, the bilateral internal thoracic arteries (ITAs) were harvested in a skeletonized fashon. The deep pericardial stitch with the gauze pad behind the heart provided an almost immobilized surgical field even when 


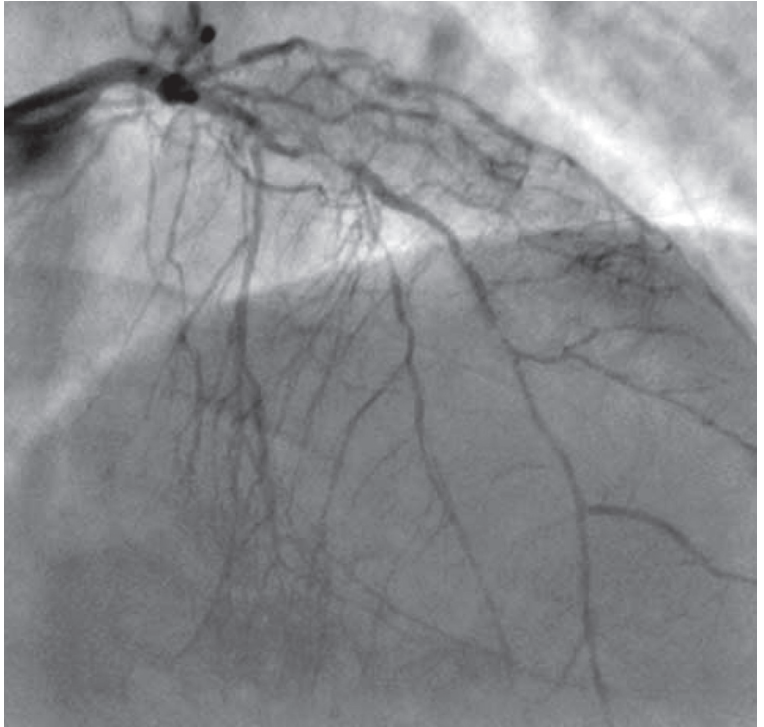

Fig. 1

a: Left coronary angiogram before percutaneous balloon angioplasty, demonstrating the diffusely diseased coronary artery with $99 \%$ stenosis. Black arrow; Pseudoaneurysm.

b: Left coronary angiogram 2 weeks after the initial intervention, revealing the pseudoanerysm formation at the inflated site.

single Octopus stabilizer was used. At first, the revascularization of circumflex area was started using the left ITA to increase the blood supply to the heart before the long onlay bypass grafting to LAD which might take longer ischemic time. Next, the arteriotomy on LAD was started at $5 \mathrm{~cm}$ distal to the aneurysm where no calcified lesion was found, then extending proximally to the aneurysm. The hemostasis was obtained by inserting the vascular probe (Synovis Life Technologies, Inc.) instead of snaring the coronary by the elastic sutures, which was difficult to occlude the severely calcified arterial lumen completely.

The aneurysm was formed at the site of disrupted calcified intima following PTCA and contained the fresh mural thrombus. The calcified and severely thickened intima was endoarterectomized from $5 \mathrm{~mm}$ proximal to the aneurysm to the distal LAD over $5 \mathrm{~cm}$, and the anerysmal wall was resected. A longitudinal incised right ITA was sutured from the proximal endoarterectomized site extending distally using 8-0 polypropylene suture along with some anchoring sutures. By resecting the calcified intima, we found that it was not difficult to suture the internal thoracic artery graft to LAD. To maintain adequate blood pressure during surgery, we administered noradrenaline at a dose of 0.03 to $0.07 \mu \mathrm{g} / \mathrm{kg}$ per min. The flow of right ITA to LAD was $58 \mathrm{ml} / \mathrm{min}$ and left ITA to left circumflex coronary artery (LCX) was $8 \mathrm{ml} / \mathrm{min}$. The

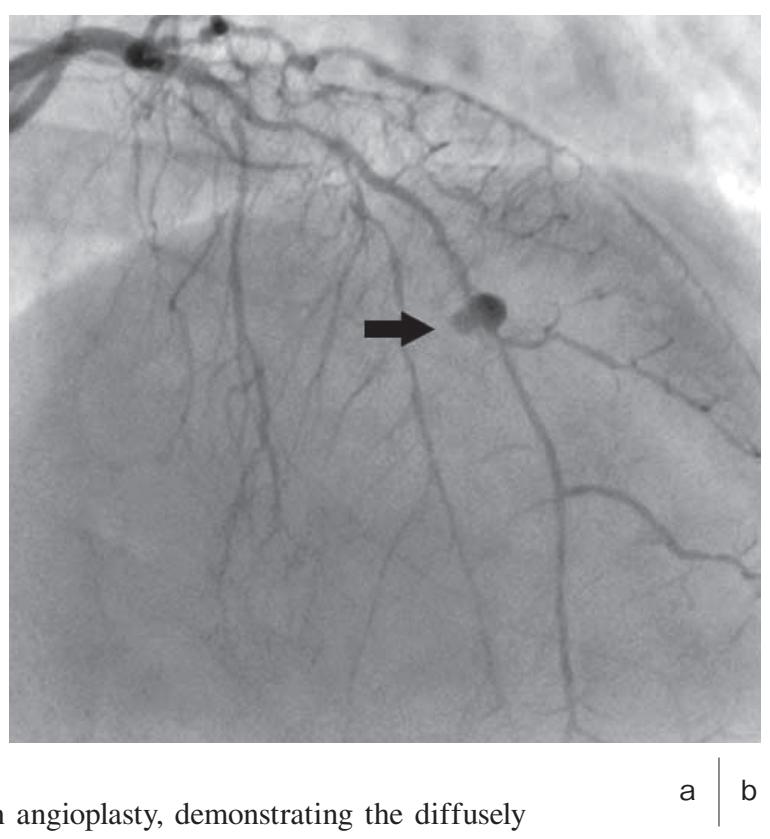

$a \mid b$ post operative course was uneventful, and she left the hospital on postoperative day 11. Now she has returned to her normal works as a farmer. The postoperative 3D CT angiogram showed the widely dilated LAD with both grafts patent and the disappearance of coronary pseudoaneurysm (Fig. 2b).

\section{Discussion}

The coronary pseudoaneurysm is a rare complication of balloon angioplasty by inflating the diseased vessels. However, the reported natural history of coronary pseudoaneurysm is favorable. Bal et al. showed only one operative case out of 28 patients who had angioplasty related aneurysm among 728 PTCA cases. ${ }^{3)}$

The operative intervention may include the rupture or rapid enlargement of the pseudoaneurysm, because there are some reports on the distal embolization or cardiac tamponade. ${ }^{4,5)}$ In our case, the rapidly expanding saccular aneurysm and the residual coronary lesions led to the surgical intervention.

The resection of pseudoaneurysm with long onlay patch without the use of cardiopulmonary bypass may induce the myocardial damage leading to the hemodynamic deterioration. However, in the case with diffusely diseased multi-vessels, the collateral circulation may have 


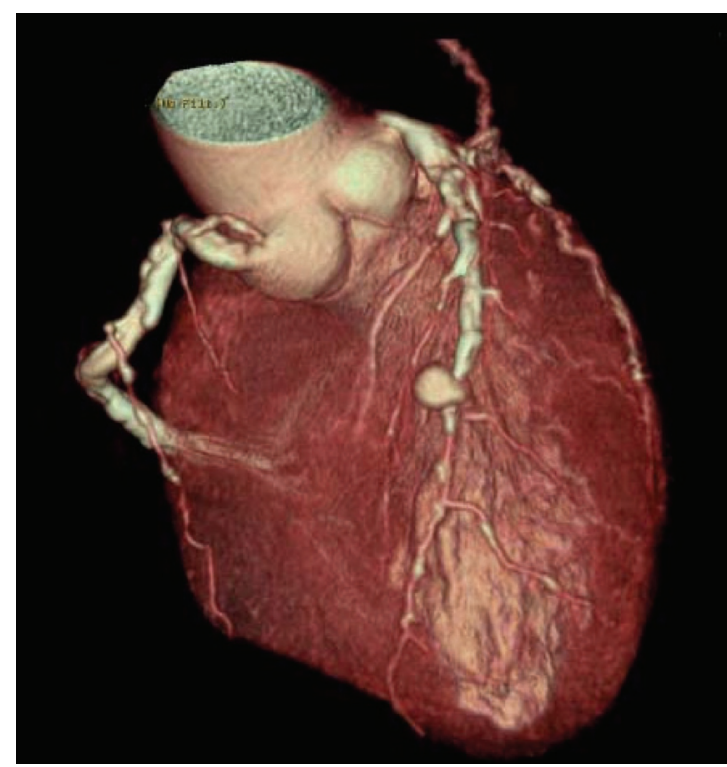

Fig. 2

a: Three dimensional coronary angiogram using computed tomography (3D CT), demonstrating the coronary aneurysm in the diffusely calcified coronary arteries.

b: 3D CT after the long onlay patch grafting using right ITA, showing the disappearance of the aneurysm and widely dilated LAD with patent both grafts.

CT, computed tomography; ITA, internal thoracic artery; LAD, left anterior descending artery developed resulting in tolerating the longer ischemic period than the isolated lesion. To avoid the ischemic disaster during the onlay patch procedure, we have revascularized the possible collateral source first before the time consuming onlay patch grafting.

In conclusion, we demonstrated in this case that the pseudoaneurysm after coronary intervention in the case of diffusely calcified lesion is safely resected using an off-pump long onlay bypass technique with the meticulous surgical strategy. Patients who have a severe diffuse coronary lesion and are a high risk for cardiopulmonary bypass will benefit from this technique.

\section{References}

1) Aqel RA, Zoghbi GJ, Iskandrian A. Spontaneous coronary artery dissection, aneurysms, and pseudoaneurysms: a review. Echocardiography 2004; 21: 175-82.

2) Lell E, Wehr G, Sechtem U. Delayed development of a coronary artery pseudoaneurysm after angioplasty. Catheter Cardiovasc Interv 1999; 47: 186-90.

3) Takanashi S, Fukui T, Hosoda Y, Shimizu Y. Off-pump long onlay bypass grafting using left internal mammary artery for diffusely diseased coronary artery. Ann Thorac Surg 23; 76: 635-7.

4) Barra J-A, Bezon E, Mondine P, Resk A, Gilard M, et al. Coronary artery reconstruction for extensive coronary disease: 108 patients and two-year follow-up. Ann Thorac Surg 2000; 70: 1541-5.

5) Saito S, Arai H, Kim K, Aoki N. Pseudoaneurysm of coronary artery following rupture of coronary artery during coronary angioplasty. Cathet Cardiovasc Diagn 1992; 26: 304-7. 